\title{
Hydroxysafflor yellow A promotes $\alpha$-synuclein clearance via regulating autophagy in rotenone-induced Parkinson's disease mice
}

\author{
Bing Han ${ }^{1}$, Lijie Wang ${ }^{2}$, Fenghua Fu², Zhenhua Wang ${ }^{1}$, Leiming Zhang ${ }^{2}$, Grace Jia $\mathbf{Q i}^{2}$, Tian Wang ${ }^{2}$ \\ ${ }^{1}$ Center of Mitochondria and Healthy Aging, School of Life Science, Yantai University, Yantai, Shandong, ${ }^{2}$ School of Pharmacy, \\ Key Laboratory of Molecular Pharmacology and Drug Evaluation (Yantai University), Ministry of Education, Collaborative Innovation \\ Center of Advanced Drug Delivery System and Biotech Drugs in Universities of Shandong, Yantai University, Yantai, Shandong, \\ PR China
}

\begin{abstract}
Autophagy is an intracellular degradation process that is involved in $\alpha$-synuclein $(\alpha$-syn) homeostasis and Parkinson's disease (PD). The purpose of this study was to investigate whether hydroxysafflor yellow A (HSYA) could promote $\alpha$-syn clearance via regulating autophagy in PD mice. Male C57BL/6 mice were intraperitoneally treated with HSYA. Thirty minutes later, they were intragastrically administered with rotenone at a dose of $30 \mathrm{mg} / \mathrm{kg}$. The hanging wire test was performed at 14 and 28 days. Then, autophagosomes and ultrastructural changes were examined by transmission electron microscopy. The expression of tyrosine hydroxylase (TH), $\alpha-s y n, J N K 1, p-J N K 1, B c l-2, p-B c l-2$, Beclin1, autophagy-related proteins (Atg) 7 and 12-5, and the LC3-II/LC3-I ratio were investigated by western blot. The hanging time of HSYA-treated $P D$ mice was significantly increased compared with that of rotenone-induced $P D$ mice $(p<0.05$ or $p<0.01)$. Compared with rotenone-induced PD mice, treatment with HSYA augmented the formation of autophagosomes. The expression of TH, p-JNK1/JNK1, Beclin1, Atg7, Atg12-5, p-BCl-2/BCl-2, and the LC3-III $L C 3-I$ ratio were significantly increased, whereas the expression of $\alpha-5 y n$ was reduced in the rotenone plus HSYA group. These results indicate that HSYA promoted $\alpha$-syn clearance via regulating autophagy in rotenone-induced PD mice.
\end{abstract}

Key words: hydroxysafflor yellow A, rotenone, neurotoxicity, $\alpha$-synuclein, autophagy.

\section{Introduction}

Over the past two decades, $\alpha$-synuclein ( $\alpha$-syn) has been shown to play a crucial role in both familial and sporadic forms of Parkinson's disease (PD) [4]. First, point mutations in the SNCA gene that encodes the $\alpha$-syn protein were demonstrated to cause PD with Lewy bodies $[16,17]$. Then, it was shown that duplication or triplication of the $\alpha$-syn locus was found in PD families and in sporadic cases [1]. Furthermore, patients with a triplication had an earlier onset, and more severe and faster progressing disease than those with duplication of the SNCA locus [2]. An important discovery was the identification of $\alpha$-syn as a component of Lewy bodies in both familial and sporadic PD patients [20]. In spo- 
radic $P D, \alpha$-syn accumulates in neuronal cell bodies and processes to form Lewy neurites in the brain, spinal cord, and peripheral nervous system [12]. To date, the exact mechanism by which $\alpha$-syn accumulation causes neuronal loss and leads to the development of PD has not been elucidated. In fact, multiple organelles have been implicated in the toxicity of $\alpha$-syn accumulation, including synaptic vesicles, mitochondria, and endoplasmic reticulum. Moreover, inter-organelle contact and organelle axonal transport are also disrupted by the toxicity of $\alpha$-syn accumulation [24].

Autophagy is a catabolic process that mediates the degradation of proteins and dysfunctional organelles in eukaryotic cells. Autophagy can be divided into three main types: macroautophagy, microautophagy, and chaperone-mediated autophagy. Macroautophagy plays a key role in the degradation of large portions of the cytoplasm, and even whole organelles, through the formation of double-membrane lipid structures called autophagosomes [10]. Autophagy is also involved in the degradation of aggregation-prone proteins, such as $\alpha$-syn. Impairment of autophagy results in $\alpha$-syn accumulation [15]. A growing body of evidence has demonstrated that there is a reduction of $\alpha$-syn degradation in PD patients [3]. It was also reported that altered autophagy is associated with a variety of neuronal diseases characterised by neurodegeneration and movement disorders [19].

The characteristic symptoms of PD have been described in ancient Chinese medical books. Chinese herbal medicine has been used to treat PD patients for centuries. Herbal medicine is still popular for the treatment of PD in Asian countries, such as China, Japan, and Korea [22]. Recent reports have demonstrated that extracts isolated from Chinese herbal medicines can modulate autophagy and elicit an improvement in neurodegenerative diseases [14]. Carthamus tinctorius L., a Chinese herbal medicine, has been widely used for the treatment cerebrovascular disease. Hydroxysafflor yellow A (HSYA) is an active component of Carthamus tinctorius L. Previous findings from our laboratory showed that HSYA could alleviate the neurotoxicity of 1-methyl-4-phenyl-1, 2, 3, 6-tetrahydropyridine by inhibiting oxidative stress [8]. HSYA improved dopaminergic neuron integrity and motor function in a rodent model of PD by increasing the brain-derived neurotrophic factor (BDNF) level in the striatum [9]. The present study aimed to inves- tigate whether HSYA promotes $\alpha$-syn clearance via regulating autophagy in rotenone-induced PD mice.

\section{Material and methods \\ Animals and HSYA}

Eight-week-old male C57BL/6 mice were acquired from Jinan Pengyue Experimental Animal Co., Ltd. (Jinan, China). Animals were housed in a climate-controlled room, maintained on a 12-h/12-h light/dark cycle, and given food and water ad libitum. The experiments were performed according to the National Institutes of Health Guidelines for the Care and Use of Laboratory Animals (publication 86-23, revised in 1986) and approved by the Institutional Animal Ethics Committee of Yantai University. HSYA (98\% purity by HPLC) was obtained from Shandong Luye Pharmaceutical Co., Ltd. (Yantai, China). HSYA is a hydrophilic compound and is stable in water. HYSA was dissolved in normal saline in the present experiment.

\section{Experimental design}

Mice were randomly divided into four groups ( $n=12$ per group): control, rotenone, HSYA, and HSYA + rotenone groups. Animals were intraperitoneally treated with either normal saline (control group and rotenone group) or $20 \mathrm{mg} / \mathrm{kg}$ HSYA (HSYA group and HSYA + rotenone group), once a day, for 28 days. Thirty minutes after each saline/HSYA administration, the rotenone and HSYA + rotenone groups were intragastrically administered with rotenone at a dose of $30 \mathrm{mg} / \mathrm{kg}$ for 28 days. The animals in the control and HSYA groups were given the same volume of $0.5 \%$ sodium carboxymethylcellulose.

\section{Hanging wire test}

The wire hanging apparatus comprised a stainless steel bar (50 cm length; $2 \mathrm{~mm}$ diameter) resting on two vertical supports and elevated $37 \mathrm{~cm}$ above a flat surface. This test was performed as previously described [18]. At 14 and $28 \mathrm{~d}$ of rotenone/HSYA treatment, mice were placed on the bar midway between the supports and were observed for $60 \mathrm{~s}$ in three trials. The amount of time spent hanging (hanging time) was recorded.

\section{Transmission electron microscopy}

The ultrastructure of autophagosomes from the striatum of animals was examined by transmission 
electron microscopy. Briefly, striatal tissues (from $n=3$ mice per group) were cut into $1-\mathrm{mm}^{3}$ sections, and fixed in $2.5 \%$ glutaraldehyde for $24 \mathrm{~h}$, postfixed with $1 \%$ osmic acid for one hour, dehydrated, and embedded. Tissues were cut into ultrathin sections of approximately $60 \mathrm{~nm}$ with a Leica EM UC7 ultra-microtome. The sections were stained with uranyl acetate and lead citrate. Then, the sections were observed using a Jeol JEM-1400 electron microscope.

\section{Western blot}

Striatal tissue was lysed in RIPA buffer and centrifuged at $12,000 \mathrm{~g}$ for $20 \mathrm{~min}$ at $4^{\circ} \mathrm{C}$. Proteins $(50 \mu \mathrm{g})$ were analysed using $8 \%$ or $10 \%$ sodium dodecyl sulphate-polyacrylamide gel electrophoresis. Then the proteins were transferred to polyvinylidene difluoride membranes at $110 \mathrm{~V}$ for $1 \mathrm{~h}$. After blocking with $5 \%$ non-fat milk for $2 \mathrm{~h}$, the membranes were incubated with the primary antibodies: rabbit anti-tyrosine hydroxylase (TH, 1 : 1000, Millipore), rabbit anti- $\alpha$ syn (1 : 1000, Millipore), rabbit anti-p-JNK1 (1 : 1000; Cell Signaling Technology), rabbit anti-JNK1 (1 : 1000; Cell Signaling Technology), rabbit anti-Beclin1 (1 : 2000; Cell Signaling Technology), rabbit anti-p-Bcl-2 (1 : 2000; Cell Signaling Technology), rabbit anti-Bcl-2 (1 : 2000; Cell Signaling Technology), rabbit anti-Atg7 (1 : 1000; Cell Signaling Technology), rabbit anti-Atg12-5 (1 :

A

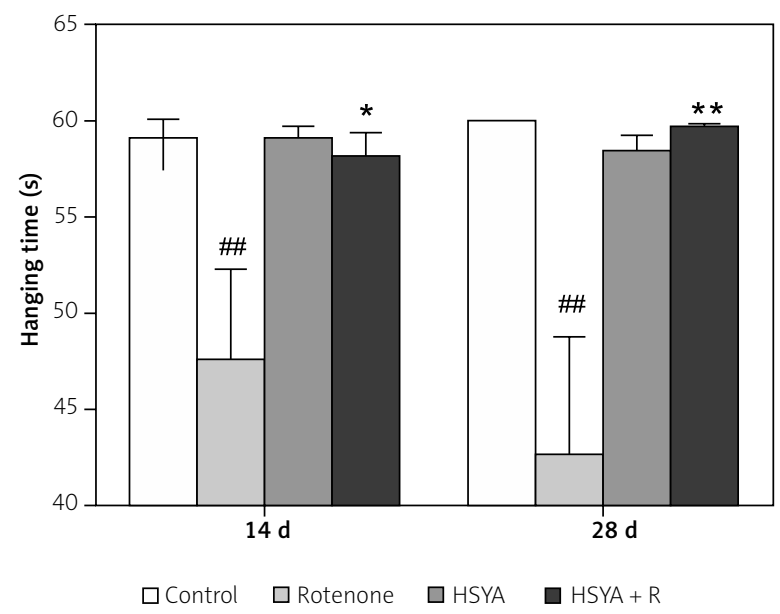

1000; Cell Signaling Technology), and mouse anti-LC3 (1 : 1000; Marine Biological Laboratory). After washing, the membranes were incubated with a horseradish peroxidase-labelled secondary antibody (mouse or rabbit, 1 : 2000; Beyotime Institute of Biotechnology). Bands were visualised using the ECL kit (Beyotime Institute of Biotechnology), and quantified using Image Quant LAS 4000 (GE Healthcare Bio-Sciences AB, Tokyo, Japan). Anti- $\beta$-actin (1 : 1000; Beyotime Institute of Biotechnology) served as the loading control.

\section{Statistical analysis}

Data were expressed as the mean \pm SEM and analysed using SPSS 20.0 Statistical Software (IBM Corp., Armonk, NY, USA). Statistical analyses were performed using one-way analysis of variance (ANOVA) followed by Tukey's test. Statistical significance was defined as $p<0.05$.

\section{Results}

\section{Effect of HSYA on the hanging time in rotenone-induced PD mice}

At 14 and $28 \mathrm{~d}$ after rotenone exposure, the hanging time of rotenone-induced PD mice was significantly decreased compared with the control group $(p<0.01)$. Compared with the rotenone group,

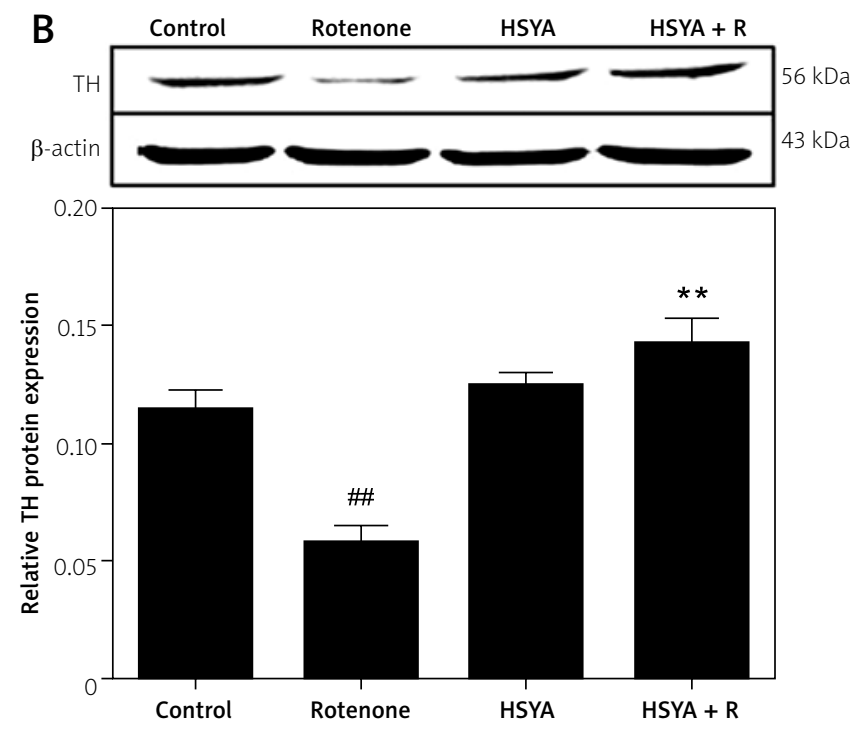

Fig. 1. Effect of hydroxysafflor yellow A (HSYA) on the hanging time $(n=12)$ and tyrosine hydroxylase $(\mathrm{TH})$ content $(n=6)$ in rotenone-induced PD mice. Data are expressed as the mean \pm SEM. Statistical analyses were performed using one-way analysis of variance (ANOVA) and Tukey's test; ${ }^{\# \#} p<0.01$ compared with control group, ${ }^{*} p<0.05,{ }^{* *} p<0.01$ compared with the rotenone group. 


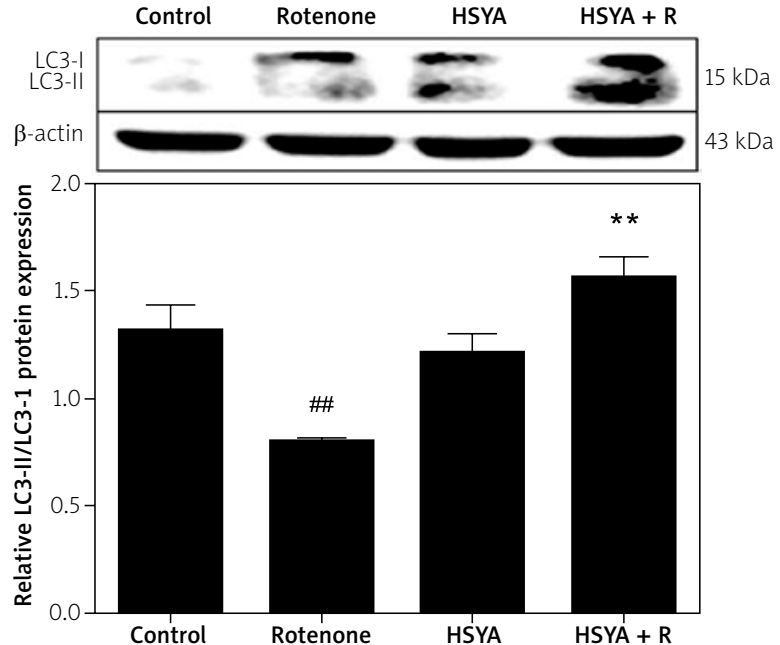

Fig. 2. Effect of hydroxysafflor yellow A (HSYA) on the LC3-II/LC3-I ratio in rotenone-induced PD mice. Data are expressed as the mean \pm SEM $(n=6)$. Statistical analyses were performed using one-way analysis of variance (ANOVA) and Tukey's test; ${ }^{\# \#} p<0.01$ compared with the control group, ${ }^{\star *} p<0.01$ compared with the rotenone group. the hanging time in the HSYA + rotenone group was significantly increased ( $p<0.05$ or $p<0.01$ ). Compared with the control group, there was no significant change in the hanging time observed for the HSYA alone group (Fig. $1 \mathrm{~A}$ ).

\section{Effect of HSYA on the TH content in rotenone-induced PD mice}

The $\mathrm{TH}$ content is shown in Fig. 1B. The TH content was decreased in the mouse striatal tissues $28 \mathrm{~d}$ after rotenone exposure. There was a significant increase in the TH content in the HSYA + rotenone group compared with the rotenone group $(p<0.01)$.

\section{Effect of HSYA on autophagy in rotenone-induced PD mice}

A significant decrease in the LC3-II/LC3-I ratio (autophagy marker) was observed in the rotenone-induced PD mice compared with the control group $(p<0.01)$. Compared with the rotenone group,
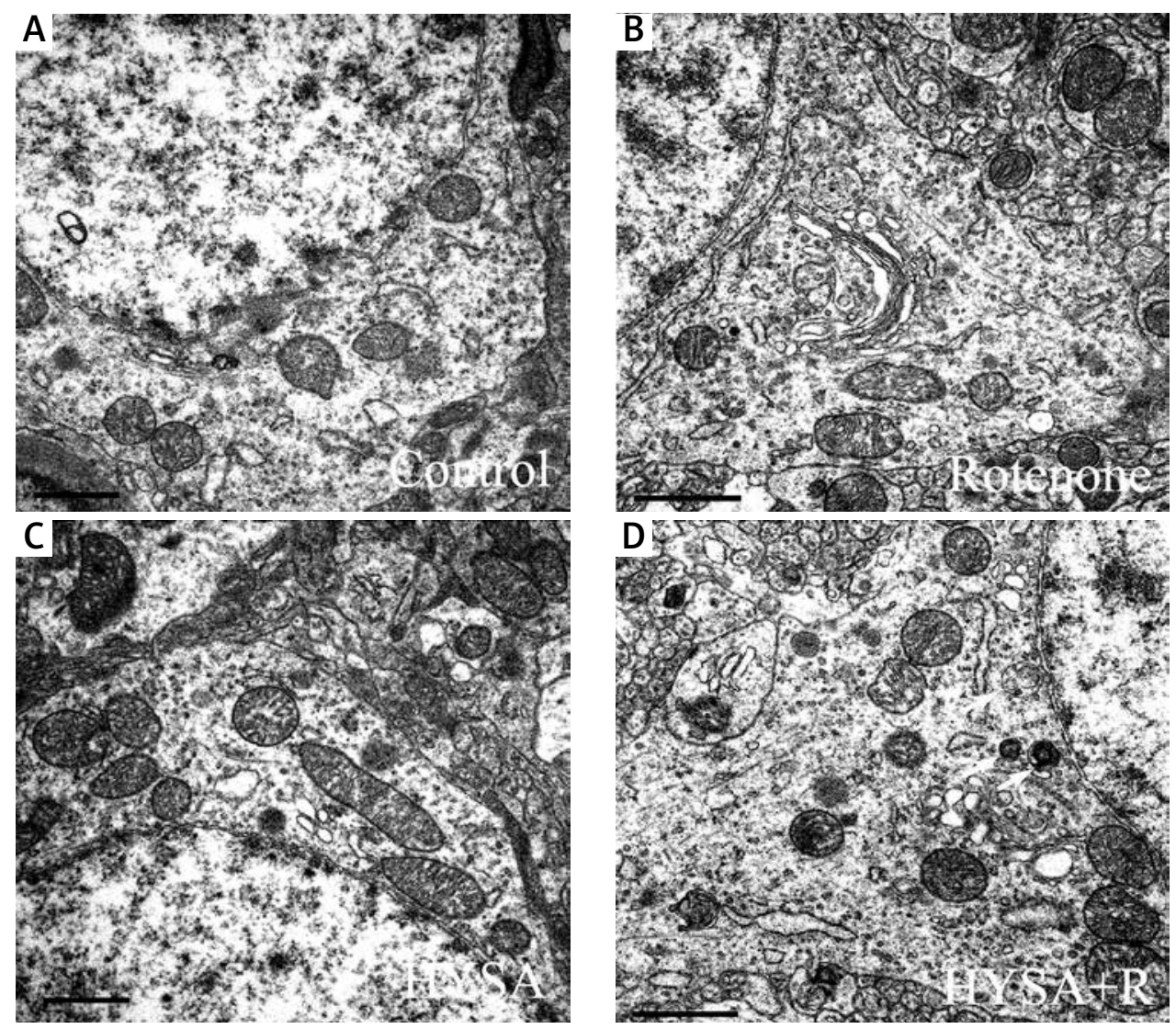

Fig. 3. Effect of hydroxysafflor yellow A (HSYA) on autophagosome formation in rotenone-induced PD mice. Typical double-membrane and lamellar autophagosomes were observed in tyrosine hydroxylase (TH) neurons of the HSYA + rotenone group. However, there were few autophagosomes observed in control, rotenone and HSYA groups $(n=3$, Scale bar $=1 \mu \mathrm{m})$. 
the HSYA + rotenone group showed a significant increase in the LC3-II/LC3-I ratio (Fig. 2, $p<0.01$ ). The LC3-II/LC3-I ratio with HSYA treatment alone was the same as that of the control group (Fig. 2). The ultrastructure of autophagosomes was investigated by transmission electron microscopy (Fig. 3). There were typical double-membrane and lamellar autophagosomes in TH neurons of the HSYA + rotenone group, consistent with the LC3-II/LC3-I ratio.

\section{Effect of HSYA on the $\alpha$-syn content in rotenone-induced PD mice}

The $\alpha$-syn content in the rotenone group increased markedly compared with that of the control group ( $p<0.01$ ). However, treatment with HSYA attenuated the augmented $\alpha$-syn content induced by rotenone ( $p<0.01$ ). Compared with the control group, there was no significant difference in the $\alpha$-syn content of the HSYA alone group, indicating that the HSYA had no effect on the $\alpha$-syn content in normal mice (Fig. 4).

Effect of HSYA on the levels of p-JNK1, p-Bcl-2, Beclin1, ATG7, and ATG12-5 in rotenone-induced PD mice

Using western blot analysis, we examined the effect of HSYA on the levels of p-JNK1, p-Bcl-2, Beclin1, ATG7, and ATG12-5 in rotenone-induced PD mice (Fig. 5). HSYA administration alone had no effect on the levels of these proteins when compared with their levels in mice of the control group. Compared with the control group, the levels of $p$-JNK1, p-Bcl-2, Beclin1, ATG7, and ATG12-5 in the rotenone group were significantly decreased $(p<0.01)$, whereas treatment with HSYA blocked their decrease $(p<0.05$ or $p<0.01)$.

\section{Discussion}

The present data suggest that HSYA can promote $\alpha$-syn clearance via regulating autophagy in a rotenone-induced PD model; $\alpha$-syn is a protein implicated in PD and thought to be one of the main pathological drivers of the disease. Based on the present results, it is reasonable to speculate that the promotion of autophagy followed by an increase in $\alpha$-syn clearance is one of the mechanisms underlying the neuroprotective effect of HSYA.

Various methods of evaluating motor impairment in PD models have been demonstrated, including catalepsy, locomotion reduction, and postural insta- bility. The hanging wire test is a sensitive method for evaluating balance, coordination, and strength in PD animals [21]. In our study, impaired hanging wire performance was observed at $14 \mathrm{~d}$ and $28 \mathrm{~d}$ of rotenone exposure. $\mathrm{TH}$ is the rate-limiting enzyme in the synthesis of dopamine. A decrease in TH levels parallels the loss of dopaminergic neurons in PD patients [7]. The present findings showed that the $\mathrm{TH}$ levels in mice of the rotenone group decreased compared with the control group. Treatment with HSYA not only ameliorated the impaired hanging wire performance but also abated the reduction of $\mathrm{TH}$ level in the rotenone-induced PD model. These results suggested that HSYA can attenuate the injury to dopaminergic nerves and therefore improve the motor function of rotenone-induced PD mice.

Most assays for autophagy use LC3 as a substrate to measure autophagic flux. LC3 can be cleaved by Atg4 to form LC3-I. Then, LC3-I conjugates to phosphatidylethanolamine to form LC3-II. LC3-II is localised to isolation membranes and autophagosomes [11]. The LC3-II/LC3-I ratio is the most widely used autophagosome marker because it reflects the number of autophagosomes and autophagy-related

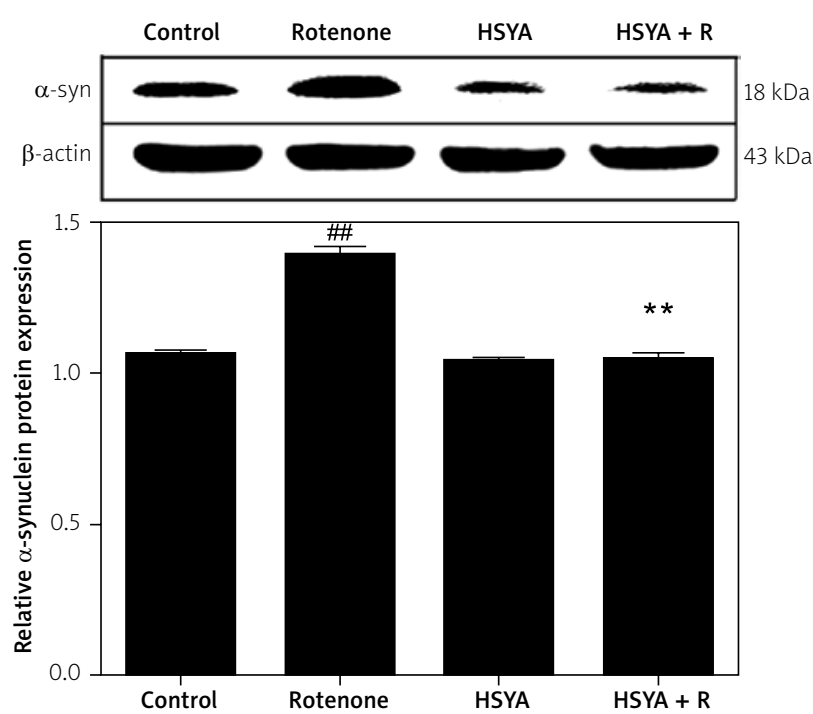

Fig. 4. Effect of hydroxysafflor yellow A (HSYA) on the $\alpha$-synuclein content in rotenone-induced PD mice. Data are expressed as the mean \pm SEM $(n=6)$. Statistical analyses were performed using one-way analysis of variance (ANOVA) and Tukey's test; ${ }^{\# \#} p<0.01$ compared with the control group. ${ }^{\star *} p<0.01$ compared with the rotenone group. 

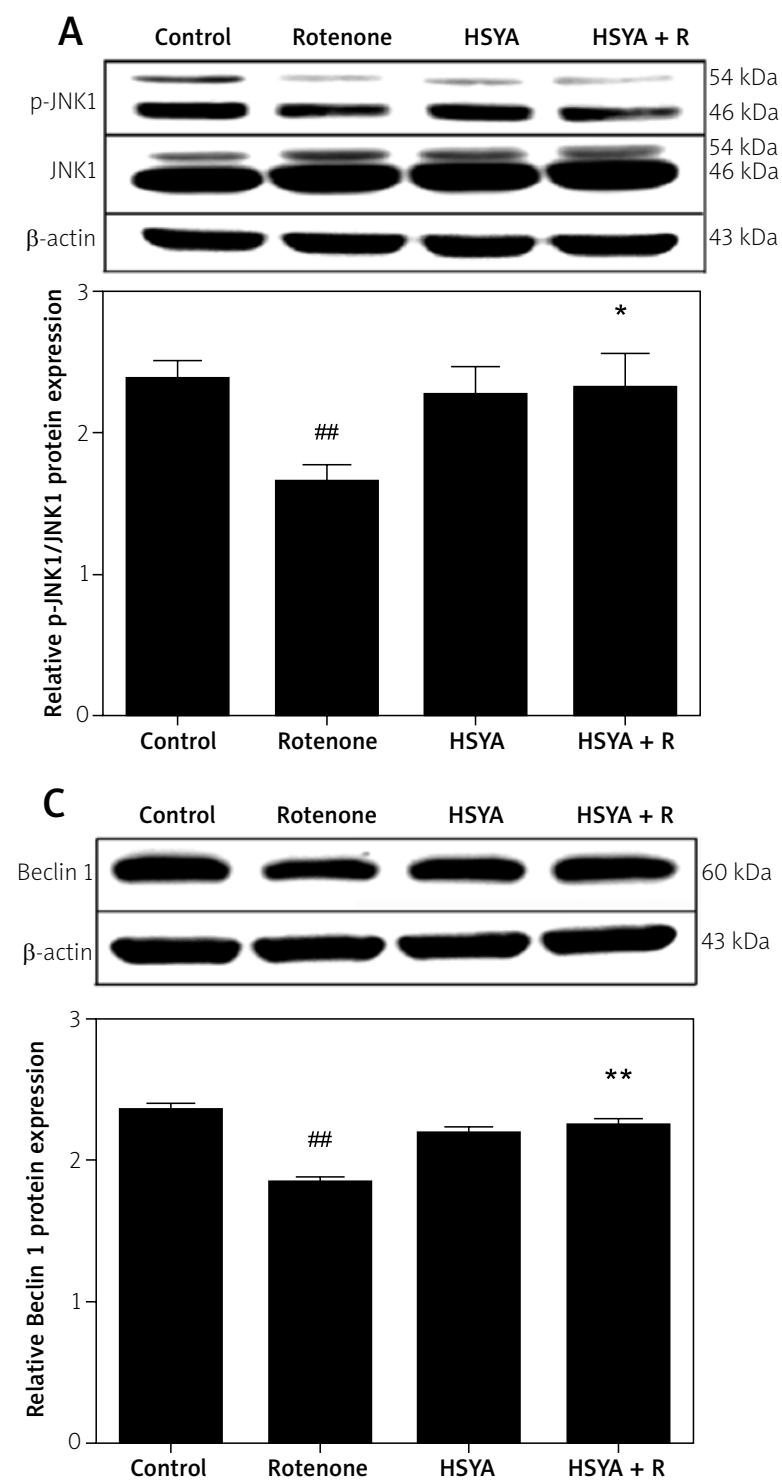

Fig. 5. Effect of HSYA on the levels of p-JNK1, p-Bcl-2, Beclin1, ATG7, and ATG12-5 in rotenone-induced PD mice. Data are expressed as the mean \pm SEM $(n=6)$. Statistical analyses were performed using one-way analysis of variance (ANOVA) and Tukey's test; ${ }^{\# \# p}<0.01$ compared with the control group, ${ }^{*} p<0.05$, ${ }^{* *} p<0.01$ compared with the rotenone group.
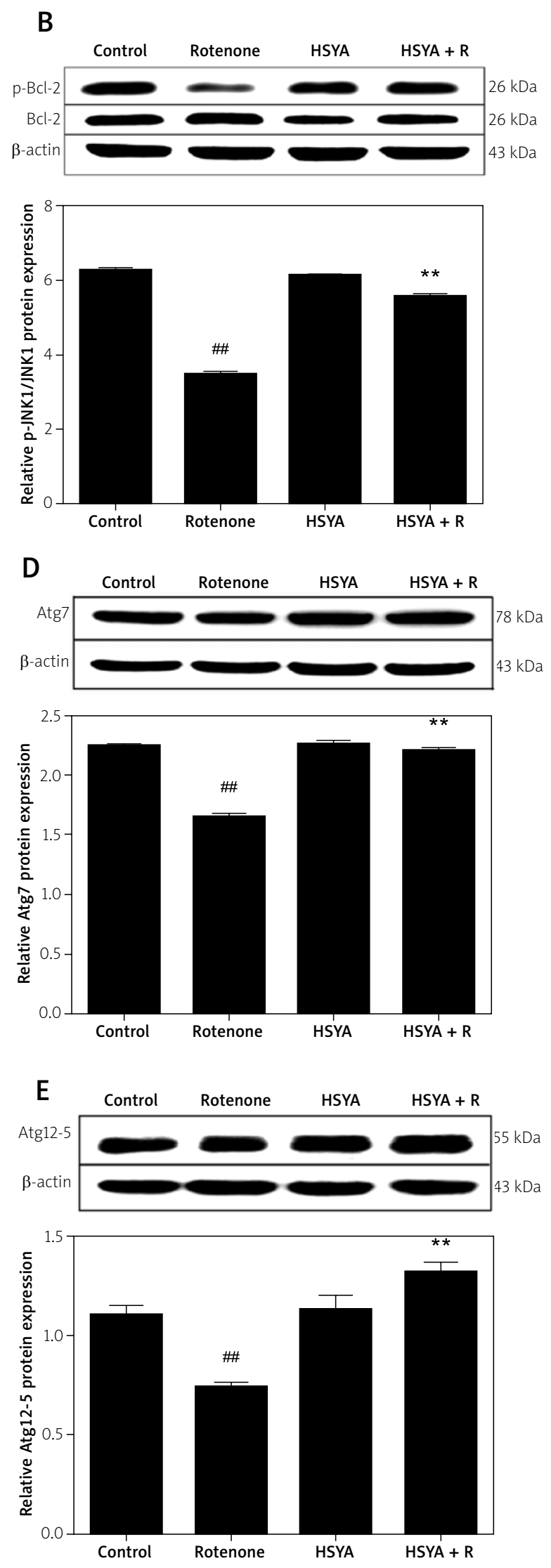
structures [6]. The increased LC3-II/LC3-I ratio and the existence of autophagosomes in the present study confirmed that HSYA enabled autophagosome formation in the rotenone-induced PD mice. The accumulation of $\alpha$-syn promotes inclusion formation, which leads to the formation of Lewy bodies. One possible strategy to prevent PD is to decrease the soluble $\alpha$-syn level. A previous study showed that autophagy plays a key role in $\alpha$-syn degradation [5]. Accompanying the augmentation of autophagy, HSYA treatment reduced the $\alpha$-syn level in rotenone-induced PD mice. These results indicate that HSYA can promote $\alpha$-syn clearance via regulating autophagy.

The JNK1 signalling pathway has also been shown to regulate autophagy [13]. In mammalian cells, Bcl-2 binds to Beclin1 and inhibits the autophagy function of Beclin1. JNK1 activation increases $\mathrm{BCl}-2$ phosphorylation, then $\mathrm{Bcl}-2$ dissociates from Beclin 1 and stimulates autophagy by disrupting the Bcl-2/Beclin1 complex [23]. These findings are consistent with our observation that HSYA augmented the levels of p-JNK1 and p-Bcl-2, which would lead to the release of Beclin 1 and an increase of autophagy-related proteins (ATG7, ATG12-5).

In summary, the present study suggested that HSYA can promote $\alpha$-syn clearance via regulating autophagy in a PD model. The mechanism of pharmacological action of HSYA at least partly involved JNK1-mediated phosphorylation of Bcl-2, followed by disruption of the Bcl-2/Beclin1 complex (Fig. 6).

\section{Acknowledgements}

This work was supported by the Joint Fund Project of the Education Department of Shandong Province (NO. ZR2014JL052), the Taishan Scholar Project (For Prof. Rong Zhai), and the 2016 Yantai ShuangBai Scholar Program. We thank Ann Turnley, PhD, from Edanz Group (www.edanzediting.com/ac) for editing a draft of this manuscript.

\section{Disclosure}

The authors report no conflict of interest.

\section{References}

1. Ahn TB, Kim SY, Kim JY, Park SS, Lee DS, Min HJ, Kim YK, Kim SE, Kim JM, Kim HJ, Cho J, Jeon BS. alpha-Synuclein gene duplication is present in sporadic Parkinson disease. Neurology 2008; 70: 43-49.

2. Chartier-Harlin MC, Kachergus J, Roumier C, Mouroux V, Douay X, Lincoln S, Levecque C, Larvor L, Andrieux J, Hulihan M, Wauc-

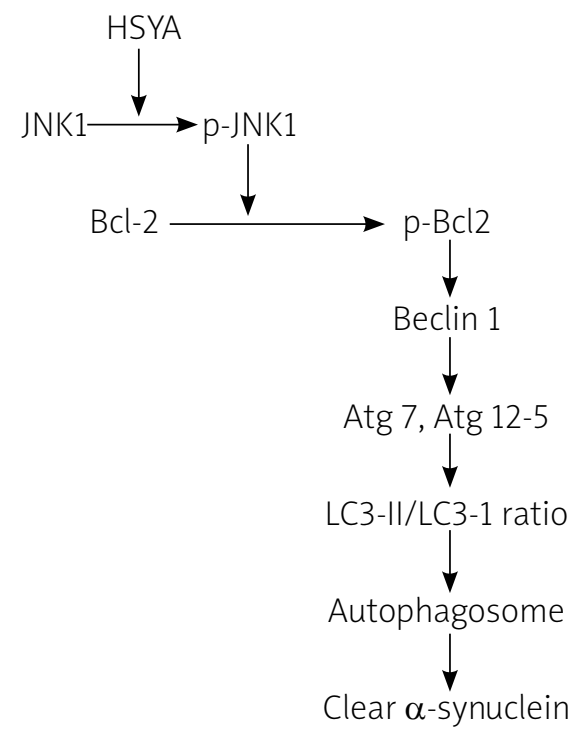

Fig. 6. Schematic diagram of hydroxysafflor yellow A (HSYA) promoting $\alpha$-synuclein clearance via regulating autophagy in rotenone-induced Parkinson's disease mice.

quier N, Defebvre L, Amouyel P, Farrer M, Destée A. Alphasynuclein locus duplication as a cause of familial Parkinson's disease. Lancet 2004; 364: 1167-1169.

3. Dehay B, Martinez-Vicente M, Caldwell GA, Caldwell KA, Yue Z, Cookson MR, Klein C, Vila M, Bezard E. Lysosomal impairment in Parkinson's disease. Mov Disord 2013; 28: 725-732.

4. Dehay B, Bourdenx M, Gorry P, Przedborski S, Vila M, Hunot S, Singleton A, Olanow CW, Merchant KM, Bezard E, Petsko GA, Meissner WG. Targeting alpha-synuclein for treatment of Parkinson's disease: mechanistic and therapeutic considerations. Lancet Neurol 2015; 14: 855-866.

5. Engelender S. alpha-Synuclein fate: proteasome or autophagy? Autophagy 2012; 8: 418-420.

6. Fan B, Zhang X, Ma Y, Zhang A. Fangchinoline Induces Apoptosis, Autophagy and Energetic Impairment in Bladder Cancer. Cell Physiol Biochem 2017; 43: 1003-1011.

7. Haavik J, Toska K. Tyrosine hydroxylase and Parkinson's disease. Mol Neurobiol 1998; 16: 285-309.

8. Han B, Zhao H. Effects of hydroxysafflor yellow A in the attenuation of MPTP neurotoxicity in mice. Neurochem Res 2010; 35: 107-113.

9. Han B, Hu J, Shen J, Gao Y, Lu Y, Wang T. Neuroprotective effect of hydroxysafflor yellow A on 6-hydroxydopamine-induced Parkinson's disease in rats. Eur J Pharmacol 2013; 714: 83-88.

10. Hurley JH, Young LN. Mechanisms of Autophagy Initiation. Annu Rev Biochem 2017; 86: 225-244.

11. Kabeya Y, Mizushima N, Ueno T, Yamamoto A, Kirisako T, Noda T, Kominami E, Ohsumi Y, Yoshimori T. LC3, a mammalian homologue of yeast Apg8p, is localized in autophagosome membranes after processing. EMBO J 2000; 19: 5720-5728.

12. Kalia LV, Lang AE. Parkinson's disease. Lancet 2015; 386: 896912. 
13. Lu B, Capan E, Li C. Autophagy induction and autophagic cell death in effector T cells. Autophagy 2007; 3: 158-159.

14. Meng X, Wang M, Sun G, Ye J, Zhou Y, Dong X, Wang T, Lu S, Sun X. Attenuation of Abeta25-35-induced parallel autophagic and apoptotic cell death by gypenoside XVII through the estrogen receptor-dependent activation of Nrf2/ARE pathways. Toxicol Appl Pharmacol 2014; 279: 63-75.

15. Moors T, Paciotti S, Chiasserini D, Calabresi P, Parnetti L, Beccari T, van de Berg WD. Lysosomal Dysfunction and alpha-Synuclein Aggregation in Parkinson's Disease: Diagnostic Links. Mov Disord 2016; 31: 791-801

16. Polymeropoulos MH, Lavedan C, Leroy E, Ide SE, Dehejia A, Dutra A, Pike B, Root H, Rubenstein J, Boyer R, Stenroos ES, Chandrasekharappa S, Athanassiadou A, Papapetropoulos T, Johnson WG, Lazzarini AM, Duvoisin RC, Di lorio G, Golbe LI, Nussbaum RL. Mutation in the alpha-synuclein gene identified in families with Parkinson's disease. Science 1997; 276: 2045 2047.

17. Pasanen P, Myllykangas L, Siitonen M, Raunio A, Kaakkola S, Lyytinen J, Tienari PJ, Pöyhönen M, Paetau A. Novel alpha-synuclein mutation A53E associated with atypical multiple system atrophy and Parkinson's disease-type pathology. Neurobiol Aging 2014; 35: 2180 e2181-2185.

18. Palomo-Garo C, Gomez-Galvez Y, Garcia C, Fernandez-Ruiz J. Targeting the cannabinoid $\mathrm{CB} 2$ receptor to attenuate the progression of motor deficits in LRRK2-transgenic mice. Pharmacol Res 2016; 110: 181-192.

19. Ravikumar B, Duden R, Rubinsztein DC. Aggregate-prone proteins with polyglutamine and polyalanine expansions are degraded by autophagy. Hum Mol Genet 2002; 11: 1107-1117.

20. Spillantini MG, Schmidt ML, Lee VM, Trojanowski JQ, Jakes R, Goedert M. Alpha-synuclein in Lewy bodies. Nature 1997; 388: 839-840.

21. von Wrangel C, Schwabe K, John N, Krauss JK, Alam M. The rotenone-induced rat model of Parkinson's disease: behavioral and electrophysiological findings. Behav Brain Res 2015; 279: 52-61.

22. Wang X, Liang XB, Li FQ, Zhou HF, Liu XY, Wang JJ, Wang XM. Therapeutic strategies for Parkinson's disease: the ancient meets the future - traditional Chinese herbal medicine, electroacupuncture, gene therapy and stem cells. Neurochem Res 2008; 33: 1956-1963.

23. Wei Y, Pattingre S, Sinha S, Bassik M, Levine B. JNK1-mediated phosphorylation of $\mathrm{Bcl}-2$ regulates starvation-induced autophagy. Mol Cell 2008; 30: 678-688.

24. Wong YC, Krainc D. alpha-synuclein toxicity in neurodegeneration: mechanism and therapeutic strategies. Nat Med 2017; 23: 1-13. 